\title{
Signaling silence-breaking ground and spreading out
}

\author{
Hye Ryun Woo and Eric J. Richards ${ }^{1}$ \\ Department of Biology, Washington University in St. Louis, St. Louis, Missouri 63130, USA
}

Among the persistent mysteries in epigenetics are the criteria by which specific regions of the genome are chosen for deposition of distinguishing chromatin marks. Once a particular region is modified, will the newly acquired epigenetic state spill onto neighboring regions of the genome or be confined to tidy patches? The report by Henderson and Jacobsen in the the previous issue of Genes \& Development (pp. 1597-1606) addresses these questions while providing insight into the utility of DNA methylation for a eukaryotic genome.

\section{Overview of DNA methylation}

In diverse eukaryotes, methylation of cytosine residues, catalyzed by DNA methyltransferases (DNMTs) (Goll and Bestor 2005), is an important epigenetic mechanism that affects gene expression and chromatin organization. In mammals, DNA methylation is restricted to CG dinucleotides, but DNA methylation in plants and fungi occurs at $\mathrm{CG}, \mathrm{CHG}$, and $\mathrm{CHH}$ sequences (where $\mathrm{H}$ is $\mathrm{A}$, $\mathrm{C}$, or T). DNA methylation is often classified into two types: de novo and maintenance methylation. Dnmt3a and Dnmt3b are responsible for de novo DNA methylation in mammals, which is important for the establishment of methylation patterns in early embryos, during development, and during carcinogenesis. Dnmt1 maintains DNA methylation during replication by copying the DNA methylation of the old DNA strand onto the newly synthesized strand. In Arabidopsis, DOMAINS REARRANGED METHYLTRANSFERASE2 (DRM2), a relative of mammalian Dnmt3, is responsible for de novo DNA methylation in all sequence contexts (Chan et al. 2005). CG DNA methylation is maintained by MET1 (a homolog of mammalian Dnmt1), whereas non-CG methylation is maintained by CHROMOMETHYLASE3 (CMT3) and DRM2 (Chan et al. 2005).

\section{Directing de novo methylation to specific sequences}

Although much progress has been made in understanding cytosine methylation, many details are still not fully

[Keywords: Epigenetics; DNA methylation; siRNA; gene silencing] ${ }^{1}$ Corresponding author.

E-MAIL richards@wustl.edu; FAX (314) 935-4432.

Article is online at http://www.genesdev.org/cgi/doi/10.1101/gad.1694608. understood. One unanswered question concerns the mechanisms by which de novo DNA methylation is targeted to specific sequences. DNMTs are thought to have little sequence preference beyond their core target site (CG, CHG, CHH), but recent whole-genome shotgun bisulfite sequencing data in Arabidopsis indicate that nucleotides surrounding or within the core can influence methylation occupancy (Cokus et al. 2008; Lister et al. 2008). Nonetheless, these sequence context biases cannot fully explain the genomic distribution of cytosine methylation. Additional mechanisms to target cytosine methylation to specific loci include the direct or indirect association of DNMTs with sequence-specific binding proteins (Di Croce et al. 2002; Puto and Reed 2008). Another DNMT targeting mechanism relies on the recognition, either directly or via adaptor proteins, of specific chromatin modifications, such as methylated isoforms of histone $\mathrm{H} 3$ (H3meK9 and H3meK27) (Lindroth et al. 2004; Vire et al. 2006). DNA methylation can also be targeted to specific sequences by small RNAs. RNA-directed DNA methylation ( $\mathrm{RdDM})$ has been described most thoroughly in plants (Matzke and Birchler 2005), and small RNA hits correspond to approximately onethird of the methylated loci in the Arabidopsis genome (Lister et al. 2008). It is now well established that transcriptional gene silencing can be induced by promoterdirected small RNAs in mammals (Morris 2008), but until recently it was not clear whether DNA methylation of target promoter regions could be guided by small RNAs. New evidence, however, indicates that a piwiinteracting RNA (piRNA)-guided pathway can trigger the initiation of DNA methylation of transposons in mammalian germ cells (Aravin and Bourc'his 2008; Kuramochi-Miyagawa et al. 2008). These three DNMT recruitment mechanisms (via transcription factors, chromatin modification, and small RNA) may act in parallel, and indeed might overlap. For example, it is possible that a component of RdDM acts via chromatin modifications.

Tandem repeats as targets of de novo methylation

What is the contribution of the DNA sequence itself in targeting DNA methylation? Are particular sequences or sequence configurations inherently susceptible to DNA methylation? One of the most common features of methylated regions in mammalian and plant genomes is the 
presence of tandem repeats (Hutter et al. 2006; Cokus et al. 2008). The importance of the tandem repeat organization is demonstrated by numerous examples where the presence of repeats, and in some cases repeat copy number, is correlated with the stability of DNA methylation and the associated epigenetic state (Yates et al. 1999; Stam et al. 2002; Reinhart et al. 2006; Fujimoto et al. 2008). The mechanisms at work are not clear. Tandem repeats might generate secondary structures that are good substrates for DNMT activity. Another explanation lies in the ability of tandem repeats to sustain the production of small RNA, via RNA-dependent RNA polymerization coupled with Dicer activity, in a way not possible for single-copy sequences (Martienssen 2003). However, not all tandem repeats are modified by an RdDM mechanism. For example, in the euchromatic portion of the Arabidopsis genome, more than 1000 unique tandem repeats were identified yet only about $2 \%$ of these overlapped with small RNA clusters (albeit, this frequency is higher than the frequency seen at random, nonrepeated sequences [0.24\%]) (Chan et al. $2006 b)$. Further work is required to define the full range of features that recruit $\mathrm{RdDM}$ machinery to tandem repeats.

\section{The SUPPRESSOR of drm1 drm2 cmt3 (SDC) locus: discovery of an efficient silencing target}

The recent whole-genome epigenomic profiling studies in Arabidopsis (Zhang et al. 2006; Cokus et al. 2008; Lister et al. 2008) are facilitating the definition of genomic features that attract DNA methylation. Some of the first fruits of these efforts are described in the previous issue of Genes \& Development by Henderson and Jacobsen (2008). At the center of this study is the SDC gene, which contains seven short (32-bp) tandem repeats in its upstream region. This gene first emerged as a locus of interest in the microarray-based DNA methylation profiling paper of Zhang et al. (2006) because SDC fell into the relatively rare category of "promoter methylated" genes. Moreover, transcription of this gene is silenced in wildtype plants but activated in a drm $1 \mathrm{drm} 2 \mathrm{cmt} 3$ mutant with defective non-CG methylation. Henderson and Jacobsen subsequently demonstrated that mutation of the $S D C$ gene suppresses the pleiotropic developmental phenotypes exhibited by drm1 drm2 cmt3 mutants (Chan et al. 2006a)-hence the gene's name, SUPPRESSOR of drm1 drm2 cmt3. The details emerging from the study of the $S D C$ gene highlight the complexity of epigenetic regulation in Arabidopsis, and assign new and unexpected roles to familiar pathways that were previously thought to be well understood.

An unmethylated copy of SDC introduced into a wildtype background on a transgene or by a conventional genetic cross has the ability to attract efficiently both DNA methylation and silencing. The authors used the arsenal of mutants available in Arabidopsis to dissect the molecular components and pathways responsible for establishment of cytosine methylation at this locus (Fig. 1). The initial steps of the process appear to be conven- tional. Production of 24-nucleotide small RNAs corresponding to the tandem repeats is required for DRM2mediated RdDM of the repeat region. The genetic requirements for this process (e.g., DRM2, AGO4, DCL3, NRPD1a, NRPD1b, RDR2) match expectations. However, the nature of the repeat targets themselves is unanticipated-they appear to be unrelated to transposons, in contrast to many dispersed methylated regions in the genome that are intact transposons or transposon remnants.

A more unexpected twist was uncovered when the requirements for maintenance of DNA methylation on the $S D C$ repeats were tested. Methylation in all sequence contexts, including CG, was lost in the drm 1 drm $2 \mathrm{cmt} 3$ mutant deficient for non-CG methylation machinery. Formally, this result demonstrates that CG methylation in the repeats is dependent on non-CG methylation. While the converse is often true, the role reversal seen for the $S D C$ repeats could stem from two different mechanisms. First, the Dnmt1-class CG methyltransferase MET1 might not act within the $S D C$ repeats, leaving the formation of the observed CG methylation to be carried out via de novo mechanisms, presumably by DRM2. Alternatively, efficient recruitment of MET1 might require non-CG methylation. Support for the latter hypothesis comes from the observation that CG methylation is decreased within the repeat in a met1 null mutant, while non-CG methylation is largely unaffected.

\section{Spreading of silencing at $S D C$}

The SDC locus presents another unexpected result in the form of cytosine methylation spreading beyond the borders of the tandem repeats. Whole-genome bisulfite sequencing data demonstrates that the DNA methylation in Arabidopsis is generally neatly confined within sharp borders defined by the boundaries between tandem repeats and flanking nonrepetitive sequences (Cokus et al. 2008; Lister et al. 2008). The complex fine-scale mosaic of heterochromatin and euchromatin observed in the Arabidopsis chromosome 4 "knob" domain also demonstrates that spreading of epigenetic silencing is not a general phenomenon for endogenous sequences (Lippman et al. 2004). The recent description of cytosine methylation spreading from a LINE retroposon sequence into the $B N S$ coding sequence is a recently documented exception to this general trend (Saze and Kakutani 2007).

In the case of $S D C$, cytosine methylation in both CG and non-CG contexts is observed $>1 \mathrm{~kb}$ upstream of and $\sim 350$ bp downstream from the tandem repeats. Moreover, both CG and non-CG methylation outside of the tandem repeats is lost in a met1 mutant, but the non-CG methylation within the repeat region is retained. This paring back of methylation to the repeat boundaries could be explained by invoking the precedent for CG methylation as a requisite for non-CG methylation maintenance. Yet, small RNA accumulation persists over a broad region upstream of the SDC coding region, encompassing the tandem repeats and flanking regions, despite the loss of cytosine methylation in a drm1 drm2 cmt3 mutant. 


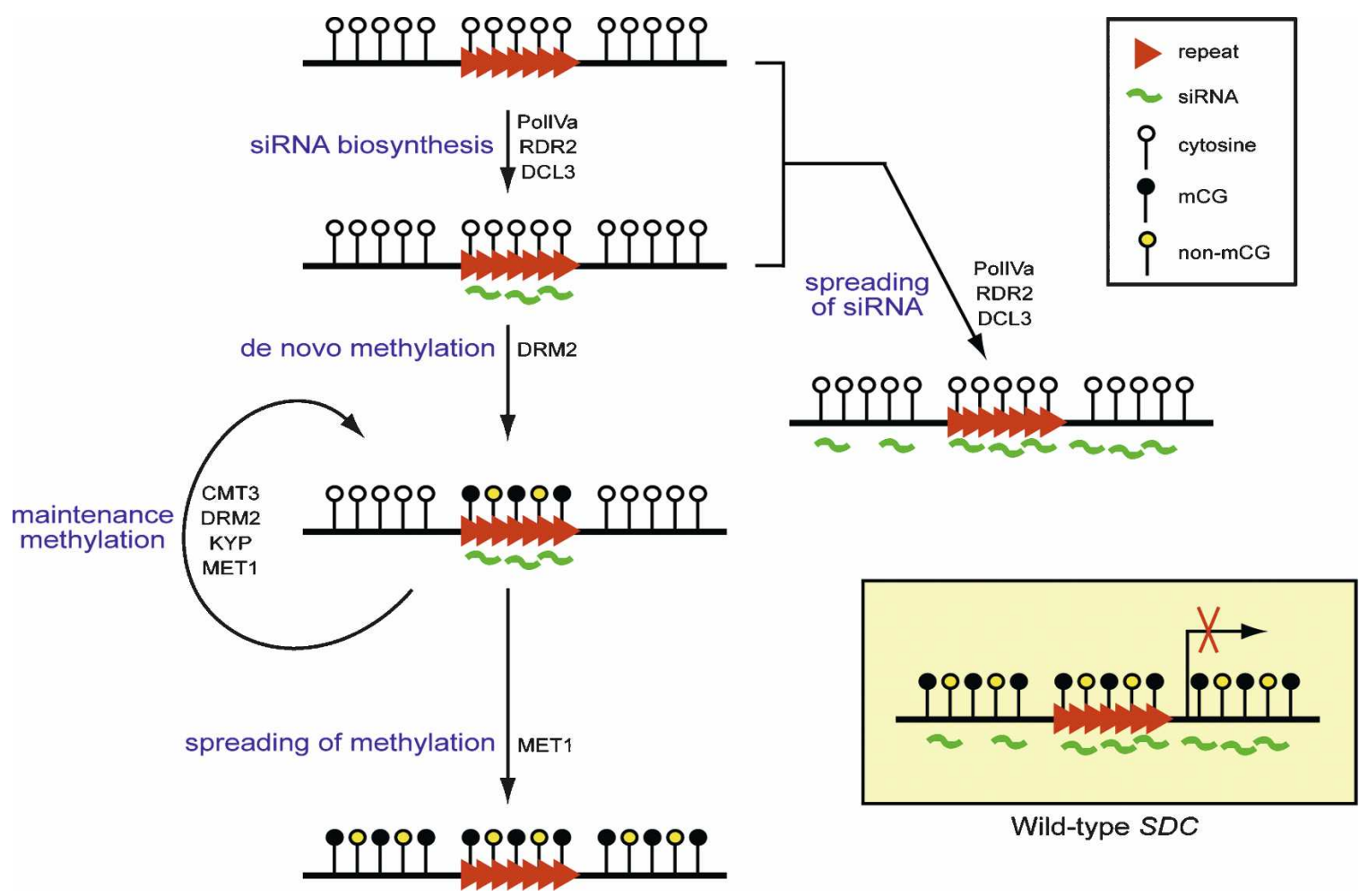

Figure 1. A model for the acquisition and maintenance of epigenetic silencing at the $S D C$ locus. The $S D C$ upstream region contains seven tandem repeats (red triangles) that recruit DNA methylation via siRNA production. The silent transcription status of $S D C$ in wild-type is accompanied by spreading of siRNAs and DNA methylation into the adjacent nonrepeated sequences (see yellow box). Establishing DNA methylation of the SDC tandem repeats requires the de novo methyltransferase DRM2 and siRNA machinery, including PolIVa, RDR2, and DCL3. DNA methylation of the repeats is maintained by CMT3, DRM2, KYP, and MET1. Spreading of DNA methylation and siRNA spreading into flanking nonrepeated sequences are independent phenomena: MET1 has a direct or indirect role in spreading of methylation of both CG and non-CG methylation; siRNA machinery is required for spreading of siRNA in a DNA methylation-independent process. However, it is not clear whether siRNA production from the tandem repeats and siRNA spreading outside of the repeats occur simultaneously or consecutively. (mCG) Methylated CG; (non-mCG) methylated non-CG; (PolIVa) DNA-dependent RNA polymerase IVa; containing NRPD1a and NRPD2a, (RDR2) RNA-DEPENDENT RNA POLYMERASE2, (DCL3) DICER-LIKE3; (CMT3) CHROMOMETHYLASE3; (DRM2) DOMAINS REARRANGED METHYLTRANSFERASE2; (KYP) KRYPTONITE; histone H3K9 methyltransferase; (MET1) METHYLTRANSFERASE1.

This result represents an apparent break in the self-reinforcing cycle of siRNA accumulation and cytosine methylation deposition (Lister et al. 2008). Moreover, small RNAs are present outside the boundaries of the repeats in the met 1 mutant, but they are not used to direct nonCG methylation-despite the presence of DRM2. This result suggests that MET1 itself might be directly responsible for methylation spreading in both CG and nonCG contexts, perhaps through an RNA-directed mechanism as first suggested for MET1 by Matzke and colleagues (Aufsatz et al. 2004).

The spreading of cytosine methylation and siRNA accumulation covers the transcription start, and expression of the $S D C$ coding sequences is inversely correlated with DNA methylation status. In wild-type Col plants $S D C$ is not transcribed but epigenetic silencing is lost to a large extent in a met1 mutant, suggesting that CG methylation in the tandem repeats and/or methylation spreading outside of the repeats is important for $S D C$ gene silencing. A more extreme abrogation of $S D C$ gene silencing occurs in drm 1 drm2 cmt3 mutants, in which both CG and non-CG are absent. The higher level of $S D C$ expression in the triple mutants is associated with abnormal phenotypes. These results suggest that nonCG methylation plays a primary role in silencing of $S D C$, but a partial role for CG methylation cannot be discounted.

\section{DNA methylation and developmental control}

To what end is all this elaborate locus-specific DNA methylation regulation directed? One answer is that cytosine methylation in organisms like plants and animals serves as an important foundation to underpin chromatin-level epigenetic codes via methylcytosine-binding proteins (Klose and Bird 2006). It is more difficult to discern the broader functional utility of DNA methylation or other epigenetic information superimposed on the genome. It was more than 30 years ago that Holliday, Pugh, and Riggs (Holliday and Pugh 1975; Riggs 1975) proposed that the addition and removal of methyl groups on cytosine could control reversible, tissue-specific gene expres- 
sion. The undeniable attractiveness of this model, augmented by many descriptions of correlations between cytosine methylation and gene expression changes has cemented the notion that DNA methylation plays a pivotal role in controlling developmental gene expression programs. The wildly diverse morphological aberrations that arise when DNA methylation is perturbed in mutants, both in plants and mammals, further supports the view that DNA methylation and developmental control are intimately associated.

These apparent connections, however, might be misleading. Bestor and colleagues (Yoder et al. 1997; Walsh and Bestor 1999) have long argued that cytosine methylation is not involved in controlling mammalian developmental gene expression programs, but rather that CG methylation in mammals is devoted to long-term transcriptional silencing of parasitic transposable elements and the reinforcement of monoallelic expression (e.g., parental imprinting, X-chromosome inactivation). The breakdown of these monoallelic expression states could account for the severe developmental phenotypes seen in mammals with defective CG methylation. The high-fidelity propagation of DNA methylation patterns at symmetrical CG sites fits well with persistent stable epigenetic states (Laird et al. 2004; Genereux et al. 2005). The existence in plants of non-CG methylation systems that are dynamic and efficiently reset using small RNA and histone modification signals raises the possibility that this category of cytosine methylation might have a direct role in executing developmental gene expression (Chan et al. 2006a) — a role that the more intransient, less labile CG methylation could not fulfill. Moreover, genome-wide cytosine methylation and expression profiling using Arabidopsis mutants demonstrate that defective non-CG methylation is frequently associated with alterations in the expression of genes, while CG methylation is generally tied to silencing of transposons and pseudogenes (Zhang et al. 2006).

It is telling then that the phenomena that Henderson and Jacobsen (2008) have uncovered at the core of the pleiotropic developmental defects in Arabidopsis mutants with crippled non-CG methylation closely parallel the situation in CG methylation mutants. The aberrant phenotypes in both cases are tied to the misexpression of remarkably few loci. The data presented by Henderson and Jacobsen (2008) demonstrate that SDC is the major locus responsible for the manifestation of developmental phenotypes in non-CG methylation mutants. The major loci involved in CG methylation mutant phenotypes include BNS and SUP, which become ectopically methylated and silenced (Jacobsen and Meyerowitz 1997; Saze and Kakutani 2007), and FWA, which loses methylation leading to inappropriate expression (Soppe et al. 2000). The important point to emphasize is that the normal regulation of BNS and SUP is not associated with cytosine methylation changes. Developmental phenotypes in bns and sup variants arise from the formation of stable hypermethylated silent epialleles. In contrast, mutant fwa epialleles are unmethylated and ectopically expressed. While FWA is a tissue-specific gene, this locus is parentally imprinted (Kinoshita et al. 2004) and therefore falls in the exceptional class of monoallelically expressed loci under DNA methylation control. Turning to the situation in non-CG methylation mutants, the question is whether the high expression level of SDC reflects a loss of DNA methylation-enforced developmental gene control or whether the unmethylated version of this gene represents another epiallele locked into an abnormal expression state. It is interesting that expression of $S D C$ is very low in wild-type plants and no tissue-specific expression has yet been detected. Further, $s d c$ insertional mutants do not exhibit abnormal developmental phenotypes. The abnormal phenotypes of non-CG methylation mutants arise from constitutive $S D C$ expression, very similar to the mechanism for $f_{w} a$ epialleles. It will be interesting to determine whether $S D C$ is another parentally imprinted locus, a constitutively silenced gene, or a locus that represents a developmentally important gene under direct DNA methylation control. Deciphering the nature of $S D C$ 's epigenetic control in wild-type plants will inform the nuanced connection between developmental perturbations in DNA methylation mutants and the true role of cytosine methylation in developmental gene control.

\section{Acknowledgments}

We thank Travis Dittmer and Hankuil Yi for comments on the manuscript. Research in E.J.R.'s laboratory is funded by grants from the National Science Foundation (MCB-0548597) and the National Institutes of Health (R01GM078256). We apologize to our colleagues whose work was not cited due to size limitations.

\section{References}

Aravin, A.A. and Bourc'his, D. 2008. Small RNA guides for de novo DNA methylation in mammalian germ cells. Genes \& Dev. 22: 970-975.

Aufsatz, W., Mette, M.F., Matzke, A.J., and Matzke, M. 2004. The role of MET1 in RNA-directed de novo and maintenance methylation of CG dinucleotides. Plant Mol. Biol. 54: 793804.

Chan, S.W., Henderson, I.R., and Jacobsen, S.E. 2005. Gardening the genome: DNA methylation in Arabidopsis thaliana. Nat. Rev. Genet. 6: 351-360.

Chan, S.W., Henderson, I.R., Zhang, X., Shah, G., Chien, J.S., and Jacobsen, S.E. 2006a. RNAi, DRD1, and histone methylation actively target developmentally important non-CG DNA methylation in Arabidopsis. PLoS Genet. 2: e83. doi: 10.1371/journal.pgen.0020083.

Chan, S.W., Zhang, X., Bernatavichute, Y.V., and Jacobsen, S.E. 2006b. Two-step recruitment of RNA-directed DNA methylation to tandem repeats. PLOS Biol. 4: e363. doi: 10.1371/ journal.pbio.0040363.

Cokus, S.J., Feng, S., Zhang, X., Chen, Z., Merriman, B., Haudenschild, C.D., Pradhan, S., Nelson, S.F., Pellegrini, M., and Jacobsen, S.E. 2008. Shotgun bisulphite sequencing of the Arabidopsis genome reveals DNA methylation patterning. Nature 452: 215-219.

Di Croce, L., Raker, V.A., Corsaro, M., Fazi, F., Fanelli, M., Faretta, M., Fuks, F., Lo Coco, F., Kouzarides, T., Nervi, C., 
et al. 2002. Methyltransferase recruitment and DNA hypermethylation of target promoters by an oncogenic transcription factor. Science 295: 1079-1082.

Fujimoto, R., Kinoshita, Y., Kawabe, A., Kinoshita, T., Takashima, K., Nordborg, M., Nasrallah, M.E., Shimizu, K.K., Kudoh, H., and Kakutani, T. 2008. Evolution and control of imprinted FWA genes in the genus Arabidopsis. PLoS Genet. 4: e1000048. doi: 10.1371/journal.pgen.1000048.

Genereux, D.P., Miner, B.E., Bergstrom, C.T., and Laird, C.D. 2005. A population-epigenetic model to infer site-specific methylation rates from double-stranded DNA methylation patterns. Proc. Natl. Acad. Sci. 102: 5802-5807.

Goll, M.G. and Bestor, T.H. 2005. Eukaryotic cytosine methyltransferases. Annu. Rev. Biochem. 74: 481-514.

Henderson, I.R. and Jacobsen, S.E. 2008. Tandem repeats upstream of the Arabidopsis endogene SDC recruit non-CG DNA methylation and initiate siRNA spreading. Genes \& Dev. 22: 1597-1606.

Holliday, R. and Pugh, J.E. 1975. DNA modification mechanisms and gene activity during development. Science 187: 226-232.

Hutter, B., Helms, V., and Paulsen, M. 2006. Tandem repeats in the CpG islands of imprinted genes. Genomics 88: 323-332.

Jacobsen, S.E. and Meyerowitz, E.M. 1997. Hypermethylated SUPERMAN epigenetic alleles in Arabidopsis. Science 277: 1100-1103.

Kinoshita, T., Miura, A., Choi, Y., Kinoshita, Y., Cao, X., Jacobsen, S.E., Fischer, R.L., and Kakutani, T. 2004. One-way control of FWA imprinting in Arabidopsis endosperm by DNA methylation. Science 303: 521-523.

Klose, R.J. and Bird, A.P. 2006. Genomic DNA methylation: The mark and its mediators. Trends Biochem. Sci. 31: 89-97.

Kuramochi-Miyagawa, S., Watanabe, T., Gotoh, K., Totoki, Y., Toyoda, A., Ikawa, M., Asada, N., Kojima, K., Yamaguchi, Y., Ijiri, T.W., et al. 2008. DNA methylation of retrotransposon genes is regulated by Piwi family members MILI and MIWI2 in murine fetal testes. Genes \& Dev. 22: 908-917.

Laird, C.D., Pleasant, N.D., Clark, A.D., Sneeden, J.L., Hassan, K.M., Manley, N.C., Vary Jr., J.C., Morgan, T., Hansen, R.S., and Stoger, R. 2004. Hairpin-bisulfite PCR: Assessing epigenetic methylation patterns on complementary strands of individual DNA molecules. Proc. Natl. Acad. Sci. 101: 204209.

Lindroth, A.M., Shultis, D., Jasencakova, Z., Fuchs, J., Johnson, L., Schubert, D., Patnaik, D., Pradhan, S., Goodrich, J., Schubert, I., et al. 2004. Dual histone H3 methylation marks at lysines 9 and 27 required for interaction with CHROMOMETHYLASE3. EMBO J. 23: 4286-4296.

Lippman, Z., Gendrel, A.V., Black, M., Vaughn, M.W., Dedhia, N., McCombie, W.R., Lavine, K., Mittal, V., May, B., Kasschau, K.D., et al. 2004. Role of transposable elements in heterochromatin and epigenetic control. Nature 430: 471-476.

Lister, R., O'Malley, R.C., Tonti-Filippini, J., Gregory, B.D., Berry, C.C., Millar, A.H., and Ecker, J.R. 2008. Highly integrated single-base resolution maps of the epigenome in Arabidopsis. Cell 133: 523-536.

Martienssen, R.A. 2003. Maintenance of heterochromatin by RNA interference of tandem repeats. Nat. Genet. 35: 213214.

Matzke, M.A. and Birchler, J.A. 2005. RNAi-mediated pathways in the nucleus. Nat. Rev. Genet. 6: 24-35.

Morris, K.V. 2008. RNA-mediated transcriptional gene silencing in human cells. Curr. Top. Microbiol. Immunol. 320: 211-224.

Puto, L.A. and Reed, J.C. 2008. Daxx represses RelB target promoters via DNA methyltransferase recruitment and DNA hypermethylation. Genes \& Dev. 22: 998-1010.

Reinhart, B., Paoloni-Giacobino, A., and Chaillet, J.R. 2006. Specific differentially methylated domain sequences direct the maintenance of methylation at imprinted genes. Mol. Cell. Biol. 26: 8347-8356.

Riggs, A.D. 1975. X inactivation, differentiation, and DNA methylation. Cytogenet. Cell Genet. 14: 9-25.

Saze, H. and Kakutani, T. 2007. Heritable epigenetic mutation of a transposon-flanked Arabidopsis gene due to lack of the chromatin-remodeling factor DDM1. EMBO J. 26: 3641-3652.

Soppe, W.J., Jacobsen, S.E., Alonso-Blanco, C., Jackson, J.P., Kakutani, T., Koornneef, M., and Peeters, A.J. 2000. The late flowering phenotype of fwa mutants is caused by gain-offunction epigenetic alleles of a homeodomain gene. Mol. Cell 6: 791-802.

Stam, M., Belele, C., Dorweiler, J.E., and Chandler, V.L. 2002. Differential chromatin structure within a tandem array 100 $\mathrm{kb}$ upstream of the maize b1 locus is associated with paramutation. Genes \& Dev. 16: 1906-1918.

Vire, E., Brenner, C., Deplus, R., Blanchon, L., Fraga, M., Didelot, C., Morey, L., Van Eynde, A., Bernard, D., Vanderwinden, J.M., et al. 2006. The Polycomb group protein EZH2 directly controls DNA methylation. Nature 439: 871-874.

Walsh, C.P. and Bestor, T.H. 1999. Cytosine methylation and mammalian development. Genes \& Dev. 13: 26-34.

Yates, P.A., Burman, R.W., Mummaneni, P., Krussel, S., and Turker, M.S. 1999. Tandem B1 elements located in a mouse methylation center provide a target for de novo DNA methylation. J. Biol. Chem. 274: 36357-36361.

Yoder, J.A., Walsh, C.P., and Bestor, T.H. 1997. Cytosine methylation and the ecology of intragenomic parasites. Trends Genet. 13: 335-340.

Zhang, X., Yazaki, J., Sundaresan, A., Cokus, S., Chan, S.W., Chen, H., Henderson, I.R., Shinn, P., Pellegrini, M., Jacobsen, S.E., et al. 2006. Genome-wide high-resolution mapping and functional analysis of DNA methylation in Arabidopsis. Cell 126: 1189-1201. 


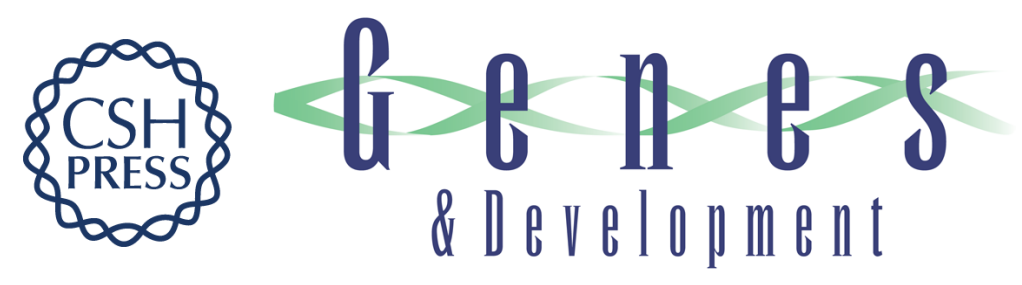

\section{Signaling silence--breaking ground and spreading out}

Hye Ryun Woo and Eric J. Richards

Genes Dev. 2008, 22:

Access the most recent version at doi:10.1101/gad.1694608

Related Content

References

This article cites 35 articles, 15 of which can be accessed free at:

http://genesdev.cshlp.org/content/22/13/1719.full.html\#ref-list-1

Articles cited in:

http://genesdev.cshlp.org/content/22/13/1719.full.html\#related-urls

\section{License}

Email Alerting

Service
Tandem repeats upstream of the Arabidopsis endogene SDC recruit non-CG DNA methylation and initiate siRNA spreading

Ian R. Henderson and Steven E. Jacobsen

Genes Dev. June , 2008 22: 1597-1606
Receive free email alerts when new articles cite this article - sign up in the box at the top right corner of the article or click here.

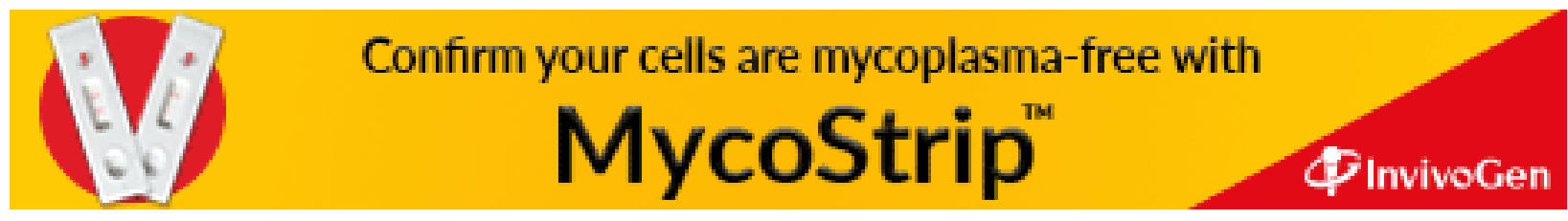

